\title{
Estudo de correlação entre o uso e cobertura da terra com a temperatura de superfície registrada pelo satélite Landsat 8
}

\author{
Correlation study between land use and cover mapping with surface \\ temperature registered by Landsat satellite 8
}

\author{
Elvis Rabuske Hendges ${ }^{1}$ \\ Franciele Ani Caovilla Follador ${ }^{2}$ \\ Juliano Andres ${ }^{3}$
}

\section{Palavras-chave:}

Sensoriamento remoto

Sensor TIRS

Processamento de imagens

Aplicativo SPRING

\begin{abstract}
Resumo
A cobertura vegetal da superfície terrestre é um dos condicionante da variação de temperatura do ar encontrado nestes locais. Assim o objetivo da presente pesquisa é verificar a correlação espacial existente entre os padrões de uso da terra e valores de temperatura de superfície (Ts) registrados pelo satélite Landsat 8. A área de estudo está localizada na zona rural de Francisco Beltrão/PR, caracterizada pela presença de usos agrícolas de subsitência. Foram processados dados de cenas datadas de 27/11/2017 e 13/12/2017, tanto para a obtenção dos mapas de uso e cobertura da terra, como para os dados de temperatura de superfície. As Ts registradas por cada uma das classes de uso da terra: água, floresta, campo sujo, campo, lavoura e solo exposto, demosntraram uma forte correlação de Pearson, sendo de 0,9116 e 0,9292 respectivamente para as cenas mapeadas. Ao analisar a homogeneidade entre as variâncias da Ts de cada classe, verificou-se que os valores das médias não possuem uma similaridade mínima significativa entre si. O teste de Tukey indicou que para a primeira data nenhuma das classes de uso apresentou valores semelhantes entre si. Já para a segunda data as classes de água e floresta apresentaram Ts estatisticamente similares, além das classes de campo sujo e campo.
\end{abstract}

Keywords:

Remote sensing

TIRS sensor

Image processing

Software SPRING

1 Universidade Estadual do Oeste do Paraná, Francisco Beltrão, Paraná, Brasil. e-mail: elvis_hendges@hotmail.com

${ }^{2}$ Universidade Estadual do Oeste do Paraná, Francisco Beltrão, Paraná, Brasil. e-mail: francaovilla@hotmail.com

${ }^{3}$ Universidade Estadual do Oeste do Paraná, Francisco Beltrão, Paraná, Brasil. e-mail: juliano.andres@gmail.com 
land use class: water, forest, dirty field, field, crop and exposed soil, showed a strong correlation of Pearson, being 0.9116 and 0.9292 respectively for the mapped scenes. When analyzing the homogeneity among the Ts variances of each class, it was verified that mean values do not have a significant minimum similarity between them. The Tukey test indicated that for the first date none of the classes of use presented values similar to each other. By the second date the water and forest classes presented Ts statistically similar, besides the classes of dirty field and field.

\section{INTRODUÇÃO}

As questões que visam estudar as consequências da relação do homem sobre a natureza de forma geral, requerem uma compreensão das relações do meio-ambiente e de sua dinâmica frente a aspectos físicos da paisagem. Sabe-se para tanto que quando o homem interfere num ambiente natural, este acaba por alterar o arranjo de elementos físicos determinantes para as condições ambientais encontradas neste local (SOARES FILHO, 1998).

Um dos elementos físicos presentes na paisagem é o clima, definido por um conjunto de variáveis (fatores e elementos) que se fundem no tempo e no espaço, revelando unidades passíveis de serem medidas (RIBEIRO, 1993). Uma das relações mais estreitas entre os fatores climáticos e as condições atmosféricas de tempo, situa-se no equilíbrio existente entre a radiação solar e a vegetação, onde elementos do clima (temperatura, umidade e pressão) são resultantes do balanço da radiação recebida e refletida para atmosfera.

Assim, a relação entre a cobertura vegetal da superfície terrestre e a temperatura do ar nestes locais se dá no controle da radiação solar (pois esta serve para reduzir a incidência dos raios sobre o solo) e da umidade do ar por meio da evapotranspiração (OLIVEIRA, 2011). Para Santos (2012), a cobertura vegetal, em especial a arbórea (através de sua folhagem mais densa), absorve a maior parte da radiação eletromagnética vinda do sol (consumida no processo de fotossíntese), impedindo que esta chegue a superfície e pela sua reflexão aqueça a camada de ar próxima. Este fato estabelece uma relação direta entre os dados da temperatura de superfície (Ts) e a temperatura atmosférica. (COELHO; RAMOS; BERGAMASCHI, 2015).

Responsável pela coleta de informações a partir da reflectancia dos objetos, o Sensoriamento Remoto é uma ciência que vem se destacando no mapeamento de alvos tanto de caráter de uso e cobertura da terra, aspectos climáticos e de condições ambientais de nosso
Planeta. A nível orbital os satélites e seus sensores são responsáveis por coletar dados e imagens impulsionando diferentes pesquisas em inúmeras áreas de outras ciências. O satélite Landsat 8, lançado pela NASA em 11 de fevereiro de 2013, opera numa altitude de 705 $\mathrm{km}$, e possui o sensor OLI (Operational Land Imager) com 9 bandas espectrais e o sensor TIRS (Thermal Infrared Sensor) com 2 bandas espectrais no infravermelho termal, bandas 10 (10.6 - $11.19 \mu \mathrm{m})$. A banda 10 do Landsat 8 pode ser utilizada após a calibração através de um modelo de transferência radiativa de emissividade e parâmetros para recuperação da temperatura da superfície, e apresenta um erro de polarização residual inferior a 2 graus para a maioria dos alvos (SCHOTT et al., 2014).

Desta forma, as informações sobre a temperatura superficial podem ser obtidas por Sensoriamento Remoto, isto porque o calor interno de um objeto é convertido em energia radiante e para a maioria dos objetos, existe uma alta correlação positiva entre a temperatura cinética verdadeira do objeto e o fluxo radiante radiado pelo objeto (JENSEN, 2009). Assim, espera-se que diferentes classes de uso apresentem distintos comportamentos termais, visto que, cada particularidade quanto aos tipos e intensidades de cobertura e uso da terra, reflete nas condições ambientais e na relação de balanço e troca de energia entre a superfície e a atmosfera.

Desta forma a presente pesquisa teve por objetivo verificar as diferenças registradas entre a Ts e as classes de uso da terra detectadas pelos sensores do satélite Landsat 8 na área rural de Francisco Beltrão/PR, Brasil.

\section{CARACTERIZAÇÃO DA ÁREA DE ESTUDO}

O município de Francisco Beltrão está situado na mesorregião sudoeste paranaense, conforme classificação do Instituto Brasileiro de Geografia e Estatística (IBGE) (Figura 1). Quanto a caracterização dos aspectos físicos de relevo, 
segundo IPARDES (2004), a mesorregião sudoeste paranaense está localizada, em toda sua extensão territorial, no Terceiro Planalto do Paraná, o qual é constituído por derrames basálticos, com cobertura sedimentar arenítica. A conformação de sua paisagem é bastante uniforme, determinada pelas formas de pequenos planaltos e patamares. O trabalho erosivo dos rios determinou a formação de um relevo de altas declividades, entre $25 \%$ e $50 \%$, em certos locais da região. Assim, o município de Francisco Beltrão apresenta o rio Marrecas como principal drenagem, sendo a área de estudo drenada pelo rio Quatorze, um dos principais afluentes da margem esquerda da bacia do Marrecas.

Figura 1 - Cartograma de localização da área de estudo.
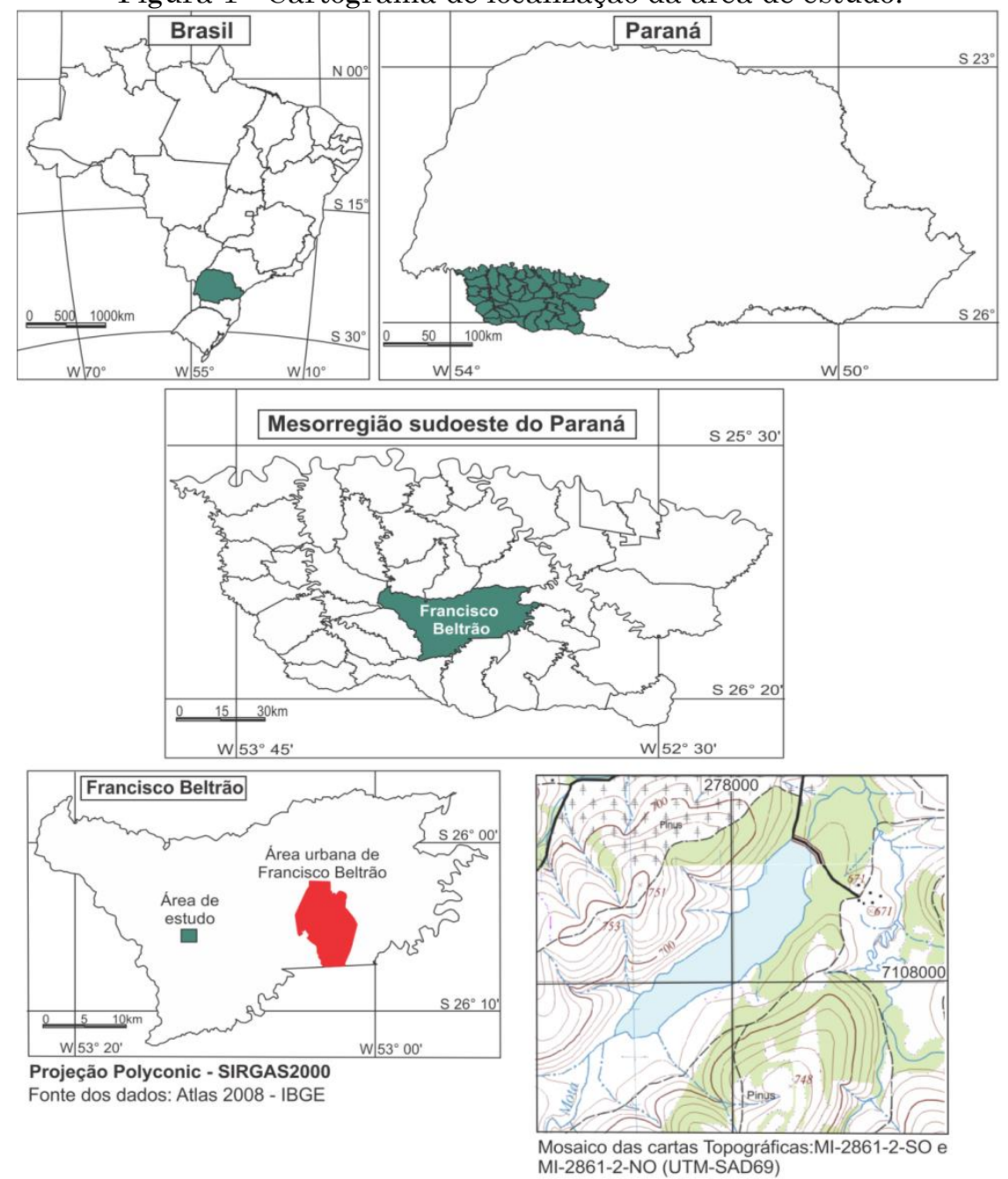

Org.: Autor, 2018.

Como aspecto climático, a área pesquisada apresenta o clima Subtropical Úmido Mesotérmico (Cfb), de verões frescos e de invernos com geadas severas e frequentes, não apresenta estação seca. As médias anuais de temperatura dos meses mais quentes são inferiores a $22^{\circ} \mathrm{C}$, e dos meses mais frios inferiores a $18^{\circ} \mathrm{C}$. As chuvas apresentam índices entre 1.600 e $1.900 \mathrm{~mm}$ precipitados e umidade relativa do ar de $85 \%$. A temperatura e a umidade são fatores determinantes na espacialização dos remanescentes nativos da vegetação característicos dos domínios fitogeográficos de Floresta Ombrófila Mista (mata de Araucária nas regiões mais elevadas) e a Floresta Estacional Semidecidual, compostas por espécies subtropicais localizada em áreas de menor altitude (IPARDES, 2004).

Quanto aos aspectos humanos a mesorregião do sudoeste paranaense é marcada pela intensa luta em torno da posse da terra, na década de 1950, tendo vistas na exploração da madeira. Atualmente os cultivos agrícolas de subsitência são a estrutura fundiária preponderante na área rural de Francisco Beltrão. No censo de 2010 (IBGE) por sua vez, contabilizou para o município uma área de $735 \mathrm{~km}^{2}$, estima-se para o ano de 2016 uma população de 87.491 habitantes 
(IBGE, 2010).

\section{MATERIAIS E MÉTODOS}

Inicialmente para o desenvolvimento da presente pesquisa foi realizada uma revisão de conceitos sobre o Sensoriamento Remoto orbital e processamento digital de imagens (ARIZA, 2013; FLORENZANO, 2005, 2007; JENSEN，2009; MOREIRA. 2005; NOVO, 1992; PONZONI; SHIMABUKURO, 2010), além do uso de imagens termais na identificação da temperatura da superfície (Ts) (COELHO; CORREA, 2013; MONTEIRO; MENDONÇA, 2003; NASCIMENTO, 2011; SOUZA; JÚNIOR, 2012).

Como fontes de dados foram utilizadas imagens do Satélite LANDSAT 8. Do sensor OLI, voltado para o mapeamento de objetos e elementos referentes ao padrão de uso e cobertura da terra. E do sensor TIRS com imagens do infravermelho termal (ARIZA, 2013). As cenas do satélite LANDSAT 8, datadas de 27 de novembro de 2017 e 13 de dezembro de 2017 foram adquirida de forma gratuita junto ao site da internet do United States Geological Survey (USGS), (https://landsat.usgs.gov/landsat-dataaccess). As características dos produtos/imagens são consistentes com o padrão de Nível 1 (ortorretificadas). $\mathrm{O}$ formato dos dados disponibilizados é um TXT (metadados) e GeoTIFFs em arquivo compactado com cerca de 1 GB (comprimido) a cerca de 2 GB (descompactado). A resolução radiométrica é de 16 Bits, Datum WGS 1984, projeção UTM (COELHO; CORREA 2013). Desta forma as imagens foram importadas para um Banco de Dados Geográfico, delimitado por coordenadas representativas da área de estudo. As bandas espectrais 1, 2, 3, 4, 5, 6 e 7 do sensor OLI foram utilizadas para realização da classificação automatizada dos pixels, transformando o número digital (ND) do registro espectral das imagens em um tema de uso da terra. Segundo Novo (1992) a interação de energia é a principal ferramenta para o estudo do sensoriamento remoto, pois é através dos diferentes padrões de resposta das interações que se pode identificar um objeto na superfície terrestre.

A fim de diminuir a confusão da matriz de classificação das imagens, foram definidas seis classes de uso da terra: água, floresta, campo sujo, campo, lavoura (áreas agrícolas em estagio inicial de crescimento das plantas, devido ao período estudado) e solo exposto (áreas agrícolas em fase de preparação para o plantio). A reflectância da vegetação é determinada por vários fatores como geometria das folhas, a morfologia, a fisiologia e a química das plantas (PONZONI; SHIMABUKURO, 2010). Estes fatores, ligados ao ângulo de incidência da luz solar permite que técnicas de processamento digital de imagens diferenciem espécies vegetais em extratos. (MOREIRA, 2005). Já o comportamento espectral das rochas e de elementos de solo exposto, é bastante semelhante, o que facilita o agrupamento de suas respostas espectrais em uma classe (JENSEN, 2009).

A classificação das imagens foi realizada no aplicativo SPRING (Sistema de Processamento de Informações Georreferenciadas) através do classificador histograma, que é algoritmo de agrupamento iterativo que classifica regiões segmentadas num determinado número prédefinido de classes de forma automática a partir da média e matriz de covariância (MOREIRA, 2011).

O nível de confiança da imagem classificada foi obtido através de uma matriz de confusão, a fim de verificar os erros oriundos do processo de atribuição dos pixels a determinadas classes. A referida matriz utilizou uma grade de pontos amostrais aleatoriamente distribuída e significativa a área classificada. A Equação 1, formula a determinação do tamanho da amostra (n) com base na estimativa da média populacional (tamanho da área).

$n=\frac{N \cdot \sigma^{2} \cdot\left(Z_{\frac{\alpha}{2}}\right)^{2}}{(N-1) \cdot(E)^{2}+\sigma^{2} \cdot\left(Z_{\frac{\alpha}{2}}\right)^{2}}$

Onde:

$\mathrm{n}=$ Número de amostras.

$\mathrm{N}$ = Tamanho da população.

$\sigma=$ Desvio Padrão populacional da variável estudada.

$\mathrm{Z} \alpha / 2=$ Valor crítico que corresponde ao grau de confiança desejado.

$\mathrm{E}=$ Margem de erro ou erro máximo de estimativa.

Com a distribuição espacial dos pontos amostrais foram elaboradas as matrizes de confusão geradas a partir da revalidação da classificação. Os pixels classificados serão comparados com imagens de altíssima resolução espacial (de até $0,5 \mathrm{~m}$ ) do satélite Pléiades $1 \mathrm{~A}$ (desenvolvido pela agência espacial francesa, CNES), disponibilizadas pelo aplicativo Google Earth. Abordagem de mapeamento elaborada por Moreira (2011), que usa como base as imagens de alta resolução espacial capturadas do Google 
Earth e técnica de interpretação visual descrita por Novo (2008).

A avaliação da acurácia foi baseada em Congalton (1991), através do cálculo do coeficiente Kappa, que leva em consideração toda matriz de confusão, inclusive os elementos de fora da diagonal principal. Os níveis de desempenho segundo o índice Kappa são: $<0=$ péssimo; $0-0,2=$ ruim; $0,2-0,4=$ razoável; 0,4 $-0,6=$ bom; $0,6-0,8=$ muito bom; e $0,8-1,0=$ excelente. Para calcular o coeficiente Kappa, foi utilizada a Equação 2.

$K=\frac{n \sum_{i-1}^{c} x_{i i}-\sum_{i-1}^{c} x_{i+} x_{+i}}{n^{2}-\sum_{i-1}^{c} x_{i+} x_{+i}}$

Onde:

$\mathrm{k}=$ coeficiente Kappa.

$\mathrm{n}=$ total de amostras.

$\sum_{i-1}^{c} x_{i i}=$ soma da diagonal principal da matriz de erro.

$\sum_{i-1}^{c} x_{i+} x_{+i}=$ somatório do produto das linhas e colunas da matriz de erro.

Para o mapeamento da Ts, Schott et al. (2014), ressalta que das imagens termais do LANDSAT 8 recomenda-se a utilização da banda 10 do somente após a calibração através de modelos de correção atmosférica e de parâmetros para recuperação da Ts, porém ainda se reconhece um erro de polarização residual inferior a 2 graus para os alvos. (SCHOTT et al. 2014).

Para o modelo de correção atmosférica das cenas do sensor termal TIRS, primeiramente foi necessário realizar o cálculo dos valores de transmissividade atmosférica e da radiância emitida e da radiância recebida pela superfície, para tal procedimento foi necessário informar dados de uma estação meteorológica local (Tabela 1), como: a temperatura do ar, a pressão atmosférica e a umidade locais na hora do registro da imagem.

Tabela 1. Dados do modelo de correção atmosférica aplicados nas imagens.

\begin{tabular}{|c|c|c|}
\hline Dados & $11 \mathrm{~h} 30 \mathrm{~min}-27 / 11 / 2017$ & 11h30min - 13/12/2017 \\
\hline Temperatura do ar ${ }^{1}$ & $22,2{ }^{\circ} \mathrm{C}$ & $24,5^{\circ} \mathrm{C}$ \\
\hline Pressão atmosférica $^{1}$ & $939,7 \mathrm{hPa}$ & $941,91 \mathrm{hPa}$ \\
\hline Umidade relativa do ar $^{1}$ & $68 \%$ & $65 \%$ \\
\hline Transmissividade atmosférica ${ }^{2}$ & $0,84 \mathrm{~K}$ & $0,82 \mathrm{~K}$ \\
\hline Radiância emitida $^{2}$ & $1,31 \mathrm{~W} / \mathrm{m}^{2} . \mathrm{sr} . \mu \mathrm{m}$ & $1,53 \mathrm{~W} / \mathrm{m}^{2} . \mathrm{sr} . \mu \mathrm{m}$ \\
\hline Radiância recebida $^{2}$ & $2,16 \mathrm{~W} / \mathrm{m}^{2} . \mathrm{sr} . \mu \mathrm{m}$ & $2,51 \mathrm{~W} / \mathrm{m}^{2} . \mathrm{sr} . \mu \mathrm{m}$ \\
\hline
\end{tabular}

Fonte: ${ }^{1}$ dados optidos do Sistema Meteorológico Paranaense (SIMEPAR) para área de estudo; ${ }^{2}$ cálculo de valores realizados pelo site da NASA http://atmcorr.gsfc.nasa.gov./

Org.: Autor, 2018.

Desta forma com os dados da transmissividade atmosférica $(0,86 \mathrm{~K})$, da radiância emitida $\left(1,10 \mathrm{~W} / \mathrm{m}^{2} . \mathrm{sr} . \mu \mathrm{m}\right)$ e recebida $\left(1,88 \mathrm{~W} / \mathrm{m}^{2} . \mathrm{sr} . \mu \mathrm{m}\right)$ pela superfície, através da Equação 3 (BARSI, 2003) foi possível efetuar o cálculo da radiância (Lt).

$L t=\frac{L_{T O A}-L_{u}-(1-\varepsilon) \cdot L_{d}}{\tau \cdot \varepsilon}$

Onde:

Lt= Radiância de um alvo negro de temperatura cinética $\left(\mathrm{W} / \mathrm{m}^{2} . \mathrm{sr} . \mu \mathrm{m}\right)$.

$\mathrm{L}_{\mathrm{TOA}}=$ Radiância espectral $\left(\mathrm{W} / \mathrm{m}^{2} \cdot \mathrm{sr} . \mu \mathrm{m}\right)$.

$\tau=$ Transmissividade atmosférica.

$\varepsilon=$ Emissividade da superfície.

$\mathrm{Lu}=$ Radiância emitida pela superfície (W/m².sr. $\mu \mathrm{m})$.

Ld = Radiância recebida pela superfície $\left(\mathrm{W} / \mathrm{m}^{2}\right.$.sr. $\left.\mu \mathrm{m}\right)$.

Como parâmetro de entrada, correspondente a emissividade, optou-se em utilizar o valor de
0,935 K, o qual corresponde ao valor médio entre superfícies sem vegetação $(0,92 \mathrm{~K})$ e áreas com vegetação (0,95 K) (NICHOL, 1994). Assim, após a correção atmosférica, foi necessário realizar o cálculo da Ts para o LANDSAT 8 a partir da conversão dos níveis de cinza da banda $10 \mathrm{em}$ radiância espectral através da Equação 4 (USGS, 2014). Com o cálculo realizado, foram ainda convertidos os valores de Ts resultantes para graus Celsius $\left({ }^{\circ} \mathrm{C}\right)$ com a subtração do valor da temperatura do ponto de congelamento da água ao nível do mar, que equivale a $273,15^{\circ}$ Kelvin.

$L_{\lambda}=M_{L} \cdot Q_{c a l}+A_{L}$

Onde:

$\mathrm{L} \lambda=$ Radiância espectral (W/m².sr. $\mu \mathrm{m})$.

$\mathrm{M}_{\mathrm{L}}=$ Fator multiplicativo de redimensionamento da banda $\left(3,3420\right.$. $\left.\mathrm{e}^{-4}\right)$.

$\mathrm{Q}_{\text {cal }}=$ Valor quantizado e calibrado do pixel em nível de cinza (DN).

$\mathrm{A}_{\mathrm{L}}=$ Fator aditivo de redimensionamento da 
banda $(0,1)$.

Os cálculos definidos pelas expressões matemáticas anteriormente mencionadas, bem como os processos de cruzamento das informações espaciais entre os mapas de uso da terra e da Ts registrados, foram realizados através da álgebra de mapas, implementada com a programação de Linguagem Espacial para Geoprocessamento Algébrico (LEGAL), no aplicativo SPRING.

Para verificar se os valores referentes a Ts registrada pelo sensor OLI condiz com a realidade terrestre encontrada, foram realizadas incursões a campo nas datas e hora da passagem do satélite pelo local imageado, onde através de um sensor de calor foram coletadas as temperaturas da água. Sabe-se que os estudos de parâmetros físico-químicos da água, em especial da temperatura, é resultado da radiação solar incidente sobre a água. As diferenças de temperatura geram camadas d'água com diferentes densidades, formando uma barreira física que impede que se misturem, e quando a energia do vento não é suficiente para misturálas, o calor não se distribui uniformemente na coluna d'água, criando assim a estratos térmicos homogêneos (ESTEVES, 1998)).

Para comparar se os dados da temperatura da água medida a campo são similares a Ts registrada pelo satélite foi realizado o cálculo estatístico teste dos sinais de amostras relacionadas. $\mathrm{O}$ teste dos sinais (não paramétrico) é aplicado em situações em que o pesquisador deseja determinar se duas condições são diferentes.

A fim de verificar estatisticamente se o fator uso e cobertura da terra, influencia no elemento climático de Ts foram cruzadas informações de cada uma das seis classes de uso, extraída uma faixa externa de $30 \mathrm{~m}$, visando diminuir a influencia de regiões caracterizadas por outros usos adjacentes.

O cruzamento supracitado originou dados para o cálculo da correlação de Pearson. O coeficiente de correlação linear de Pearson, segundo Turkman e Silva (2000), estabelece um parâmetro de medida correlativa que estabelece um nível de intensidade das relações entre as variáveis. Os resultados neste coeficiente apresentam valores num intervalo de -1 a 1 . Entende-se como correlação negativa perfeita (variáveis não possuem nenhum tipo de relação) se o valor obtido for -1 . Se o resultado do coeficiente apresentar valor 1 , a relação entre as variáveis perfeitamente direta. Para os valores intermediários, os autores indicam as seguintes análises entre os intervalos de valores: 0,01 a 0,39 a correlação é fraca; 0,40 a 0,69 a correlação é média; e 0,70 a 0,99 a correlação é forte.

Além da correlação de Pearson, foi ainda empregado o teste estatístico de Tukey e a análise de variância (ANOVA) para verificar se Ts mapeadas para cada uma das classes de uso e cobertura da terra apresentam uma diferença em sua média.

\section{RESULTADOS E DISCUSSÕES}

Após a criação do banco de dados no aplicativo SPRING, passou-se a importação das imagens do satélite LANDSAT 8. A Figura 2 apresenta as cartas imagem numa combinação falsa cor entre as bandas 7, 5 e 4 (RGB) e os mapas de uso e cobertura da terra oriundos da classificação.

Se analisada visualmente a evolução do uso e cobertura da terra da área estudada, percebe-se que inúmeras áreas classificadas como solo exposto (tons de magenta e rosa) para imagem datada de 27 de novembro de 2017 evoluíram para a classe lavoura (alterando a coloração) na imagem de 13 de dezembro de 2017. Este fato é explicado pela região ser caracterizada pelo plantio de culturas anuais como soja e milho, e no período estudado as lavouras de soja estarem numa fase inicial de crescimento.

Ao realizar a classificação das bandas, a classe de água e floresta e campo sujo, que somados representam $53 \%$ da área de estudo, apresentaram grande semelhança de área para as datas mapeadas. Já a classe lavoura apresentou um acréscimo de 38 ha (445\%), em detrimento principalmente da diminuição das áreas de solo exposto (-23 ha) e do campo (-12 ha) além do campo sujo que reduziu 03 ha.

Depois de realizada a classificação, foi realizado o cálculo que definiu os 60 pontos amostrais (10 para cada classe), utilizando-se $95 \%$ de valor para o grau de confiança e $5 \%$ como margem de erro. Assim a grade aleatória estimou uma distancia média entre pontos amostrais de $170 \mathrm{~m}$ para latitude e $330 \mathrm{~m}$ para longitude ( 6 colunas com 10 pontos cada). 
Figura 2 - Cartas imagem e mapas de uso e cobertura da terra.

Carta Imagem

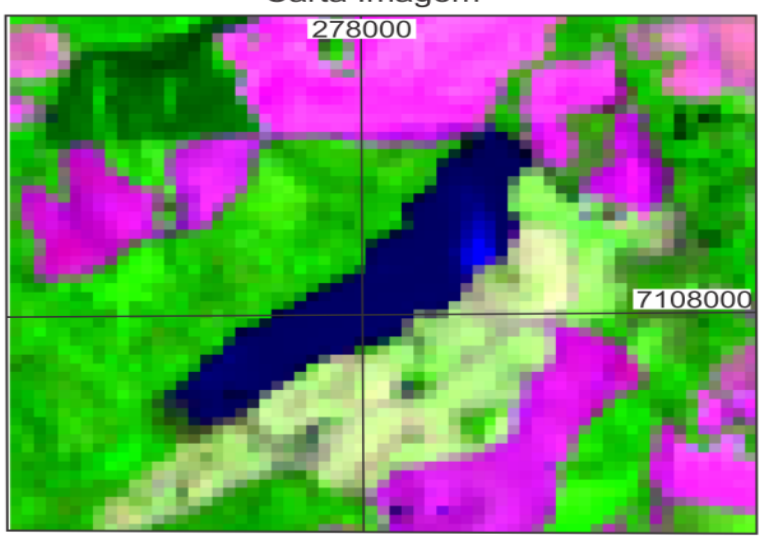

Composição colorida (RGB) bandas 7,5 e 4 respectivamente. Satélite Lnadsat 8 , sensor OLI de 27 de novembro de 2017.

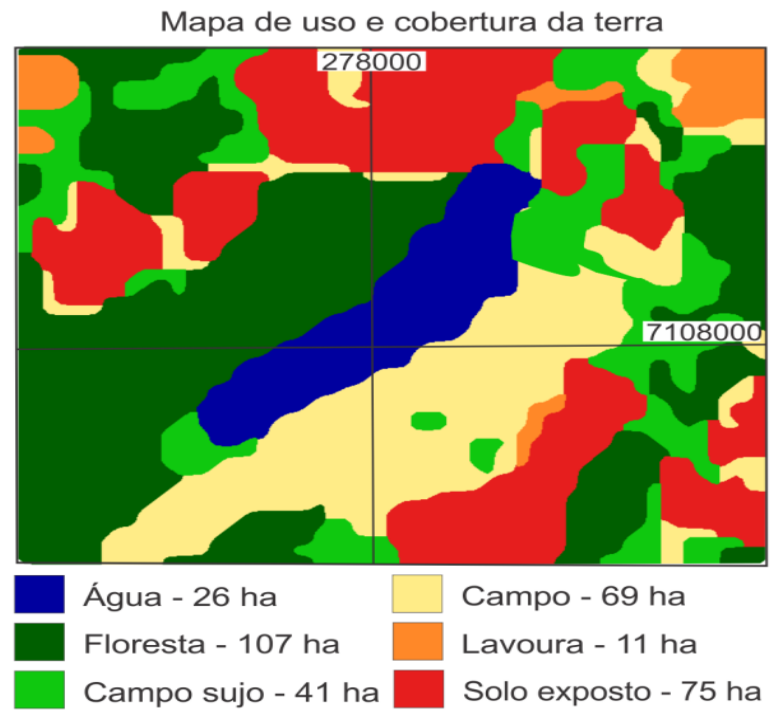

DATUM HORIZONTAL: SAD69 - MINAS GERAIS Origem da Quilometragem UTM: Equador acrescido de $10.000 \mathrm{~km}$ e Meridiano $51^{\circ} \mathrm{W} . \mathrm{GR}$. acrescidos $500 \mathrm{~km}$

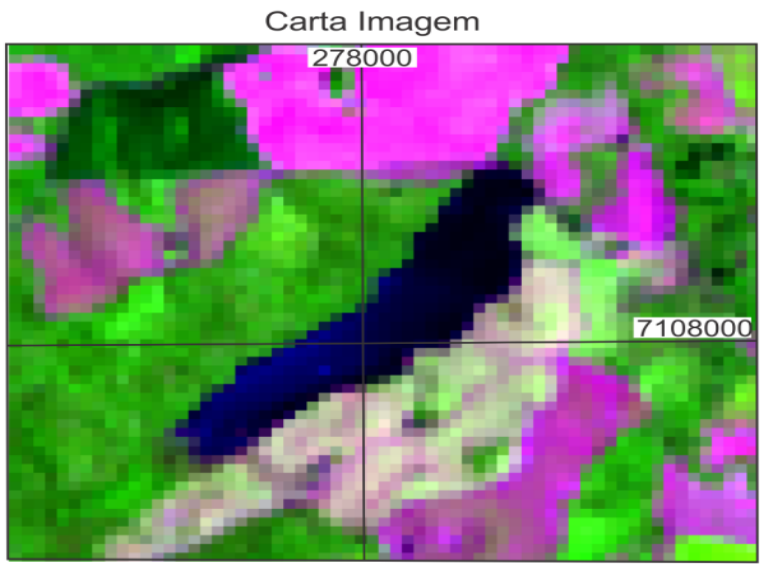

Composição colorida (RGB) bandas 7,5 e 4 respectivamente. Satélite Lnadsat 8 , sensor OLI de 13 de dezembro de 2017
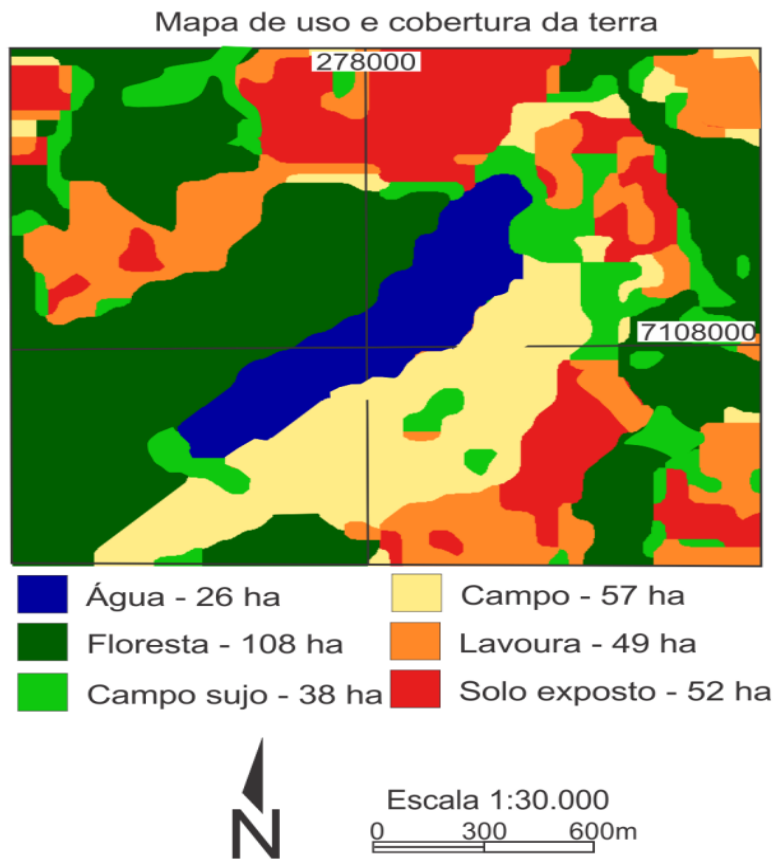

Org.: Autor, 2018.

Após comparadas as imagens classificadas (mapas de uso da terra) com a imagem do Google Earth de 01/07/2016, nos pontos amostrais prédefinidos, foi gerada uma matriz de confusão, para o cálculo do coeficiente Kappa, para analisar o grau de confiabilidade das classificações de ambas as cenas. Este cálculo resultou em valores de 0,7692 para a primeira imagem e de 0,7507 para a segunda imagem, ambos os valores enquadrados em um nível muito bom de classificação.

A imagem referente a banda 10 do sensor TIRS, após ser submetida a correção atmosférica, e ser transformada para valores de Ts em graus célsius, apresentou uma variação entre 21 à 34 ${ }^{\circ} \mathrm{C}(27 / 11 / 2017)$ e de 23 à $36{ }^{\circ} \mathrm{C}(13 / 12 / 2017)$. Cabe ressaltar que o pixel presente na imagem quantifica de Ts média registrada pelo sensor conforme a refletância dos alvos distribuídos na superfície terrestre, esta por sua vez é diferente da temperatura do ar medida pelas estações meteorológicas. Desta forma, Weng, Lu e Schubring (2004); e Coelho, Ramos e Bergamaschi (2015), em seus estudos ressaltam que a temperatura média de superfície apresenta, normalmente, valores mais elevados em relação a temperatura média do ar, porém essa diferença não infere nos resultados, uma vez, que a temperatura quantificada pelo satélite é a temperatura radiante da superfície.

A fim de verificar as informações oriundas do sensor TIRS, as Ts foram comparadas com os valores de temperaturas medidas a campo (Tabela 2), pelo teste estatístico dos sinais onde evidenciou-se uma diferença não significativa, pois $\mathrm{p}=0,096$ a $5 \%$ de significância $(\mathrm{p} \geq 0,05)$. 
Tabela 2 - Valores da temperatura verificados a campo e de Ts registrados pelo satélite.

\begin{tabular}{|c|c|c|c|c|}
\hline \multirow{2}{*}{ Ponto / Localização } & \multicolumn{2}{|c|}{$27 / 11 / 2017$} & \multicolumn{2}{|c|}{$13 / 12 / 2017$} \\
\hline & Campo $\left({ }^{\circ} \mathrm{C}\right)$ & $\operatorname{TIRS}\left({ }^{\circ} \mathrm{C}\right)$ & Campo $\left({ }^{\circ} \mathrm{C}\right)$ & $\operatorname{TIRS}\left({ }^{\circ} \mathrm{C}\right)$ \\
\hline P1 (W53¹3’02”; S2607’36”) & 22 & 23,01 & 26 & 26,15 \\
\hline P2 (W53¹3’09”; S2607’47”) & 23 & 23,24 & 26 & 25,84 \\
\hline P3 (W53¹3’19”; S2607’52”) & 23 & 23,01 & 25 & 25,33 \\
\hline P4 (W53¹3’08”; S2607’35”) & 24 & 23,75 & 25 & 24,88 \\
\hline P5 (W53¹3’15”; S2607’44”) & 24 & 23,53 & 25 & 24,64 \\
\hline P6 (W53¹3’24”; S2607’50”) & 24 & 23,42 & 25 & 24,59 \\
\hline P7 (W53¹3’22”; S2607:51”) & 24 & 23,52 & 25 & 24,55 \\
\hline P8 (W53¹3’12”; S2607’45”) & 25 & 24,88 & 25 & 24,60 \\
\hline P9 (W53¹3'5”; S2607’35”) & 25 & 24,58 & 26 & 25,24 \\
\hline
\end{tabular}

Org.: Autor, 2018.

Ao correlacionar os dados, dos mapas de uso e cobertura da terra e da Ts, foram comparados os valores de Ts de cada pixel de cada classe de uso e cobertura mapeada pelo valor da média de temperatura de cada classe. Assim o valor de correlação de Pearson apresentou valores positivos de 0,91 (correlação forte) para data de 27/11/2017, sendo que a classe água e lavoura representaram uma menor variância de suas temperaturas em relação a média da temperatura de sua classe $(\mathrm{s}=0,34$ e 0,56 respectivamente). Este fator pode ser explicado pelo extrato homogêneo, tanto para as superfícies de lâminas d'água como para culturas agrícolas em estagio inicial de crescimento. Já a classe de campo sujo, como mencionado anteriormente, composta por diferentes extratos de plantas (rasteiro, arbustivo e arbóreo) apresentou uma maior variação de temperaturas $(s=1,64)$, seguida pela classe de solo exposto com um desvio padrão de $\mathrm{s}=1,15$ (Figura 3).

Figura 3 - Gráfico de dispersão das Ts das classes de uso X Ts média de cada classes de uso para a data de $27 / 11 / 2017$.

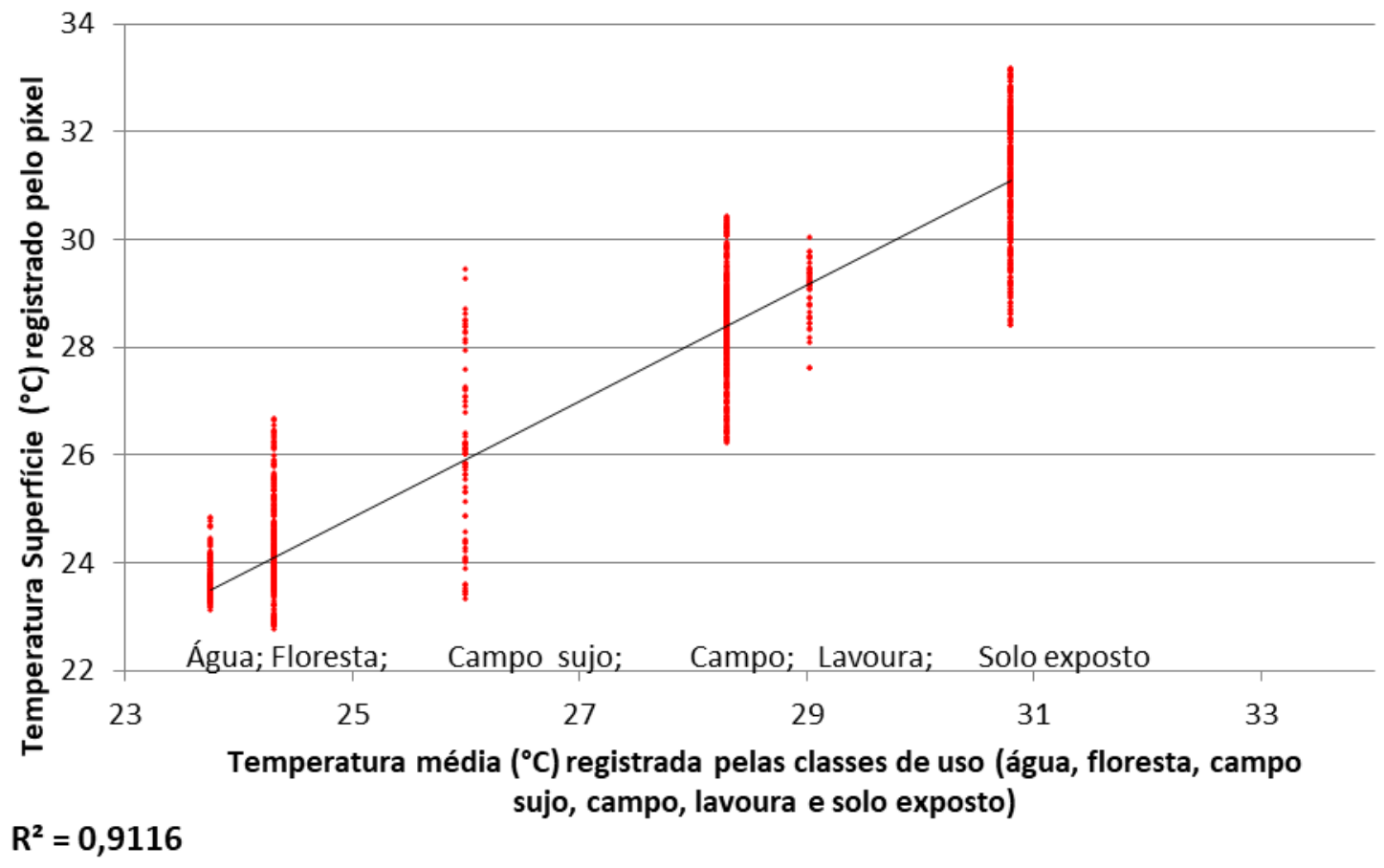

Org.: Autor, 2018.

Com médias de temperaturas superiores bem como as Ts representativas para cada pixel mapeado nas classes de uso e cobertura da terra, a data de 13/12/2017 também apresentou uma forte correlação estatística de Pearson $(0,93)$. A classe do solo exposto apresentou algumas temperaturas baixas em relação a sua média deixando esta classe com o maior desvio padrão entre as Ts medidas e a média geral da classe ( $\mathrm{s}$ $=1,41)$. Esse fator pode ser explicado pela 
umidade ou por um solo recentemente remexido pelas atividades agrícolas (Figura 4). A classe água foi a classe com menor desvio padrão para referida data com $\mathrm{s}=0,47$.

Percebe-se ainda nas figuras 3 e 4, que subtraindo a classe da água, ocorre uma tendência inversamente proporcional entre Ts e o extrato vegetal, ou seja, conforme aumenta da média da Ts identifica-se uma diminuição no extrato vegetal.

Figura 4 - Gráfico de dispersão das Ts das classes de uso X Ts média de cada classes de uso para a data de 13/12/2017.

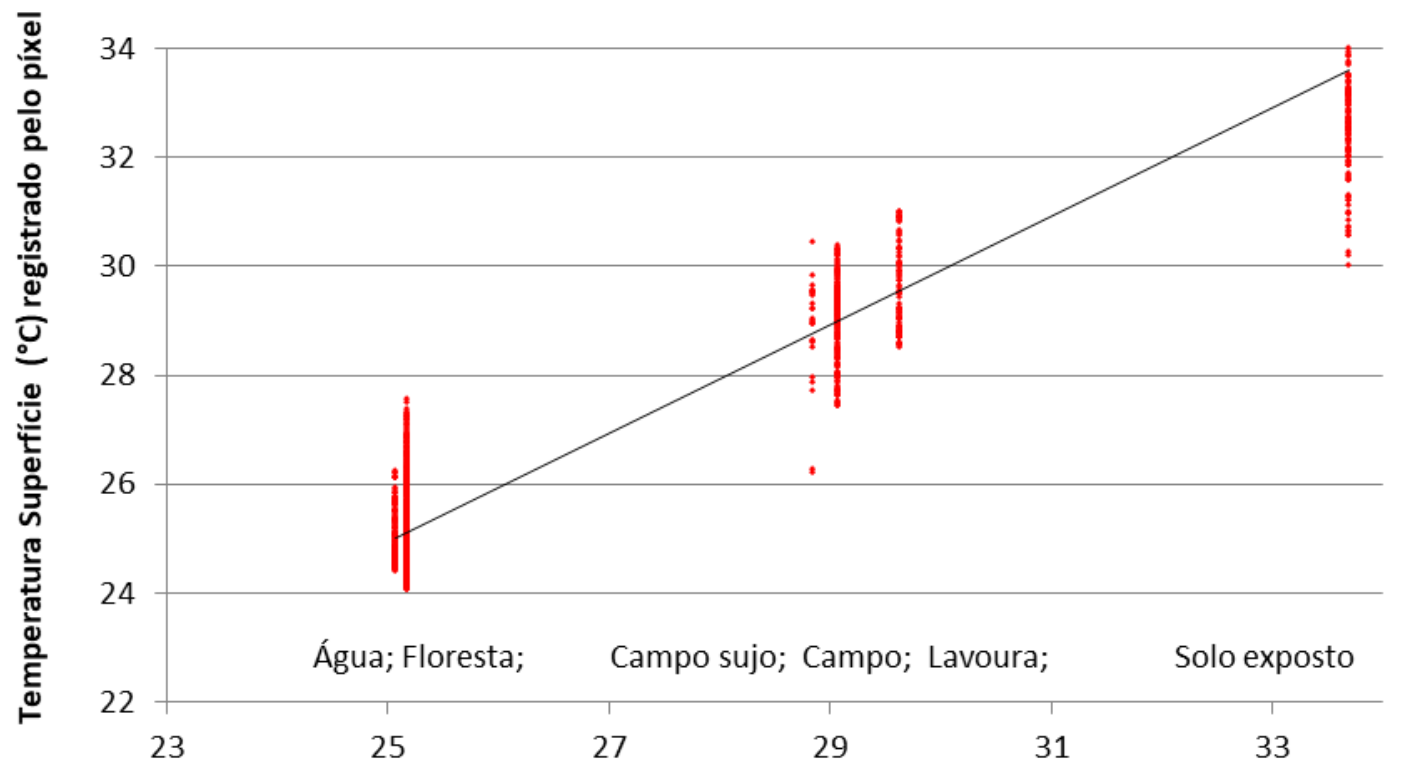

$\mathbf{R}^{2}=0,9292$ Temperatura média $\left({ }^{\circ} \mathrm{C}\right)$ registrada pelas classes de uso (água, floresta, campo
sujo, campo, lavoura e solo exposto)

Org.: Autor, 2018.

Ao analisar a homogeneidade entre as variâncias (ANOVA) para os valores da Ts de cada um dos grupos de uso e cobertura da terra nas datas estudadas o F calculado apresentou os valores de Fcal = 3.593,199 (27/11/2017) e de Fcal $=4.516,345(13 / 12 / 2017)$, com p $<0,001$ (menor que o nível de significância estabelecido de 0,05$)$, rejeitando a hipótese $\mathrm{H} 0$, ou seja, os valores das médias não possuem uma similaridade mínima significativa entre si. Já para demonstrar quais as classes de uso se diferem entre si, ou as classes que podem possivelmente ser agrupadas por semelhanças de Ts registradas, o teste de Tukey indicou que para a primeira data (27/11/2017) nenhuma das classes de uso apresentou valores semelhantes entre si. Já para a segunda data 13/11/2017 as classes de água e floresta apresentaram Ts estatisticamente similares $(0,252)$ além das classes de campo sujo e campo $(0,899)$.

\section{CONSIDERAÇÕES FINAIS}

Por meio da presente pesquisa foi possível perceber que as Ts registradas pelo sensor OLI, bem como as diferentes sensações térmicas percebidas na área rural de Francisco Beltrão/PR possuem uma relação direta com os padrões de uso da terra mapeados, principalmente levandose em conta os padrões de vegetação predominantes. Recomenda-se a realização de novos estudos com sensores capazes de operar em diferentes resoluções espectrais com comprimento do infra-vermelho termal, bem como com maiores resoluções espaciais, visto a grande variedade no mosaico de usos encontrados na área de estudo.

\section{REFERÊNCIAS}

ARIZA, A. Description y Correccion de Productos Landsat 8. Instituto Geográfico Agustin Codazzi, Bogotá, 2013.

BARSI, J, A.; BARKER, J. L.; SCHOTT, J. R. An atmospheric correction parameter calculator for a single thermal band earth-sensing instrument. In: Geoscience and Remote Sensing Symposium. Toulouse, p. 30143016, 2003.

COELHO, A. L. N.; RAMOS, A. L. D.; 
BERGAMASCHI, R. B. Aplicação dos Produtos Landsat-8/TIRS/OLI e Aster/GDEM na Avaliação do Comportamento Termal de Superfície, Usos e Topografia. XVII Simpósio Brasileiro de Sensoriamento Remoto - SBSR. João Pessoa, p. 1323-1330, 2015.

COLGATON, R. G. A review of assessing the accuracy of classifications of remotely sensed data. Remote Sensing of Environment , v. 49, n. 12, p. 1671-1678, 1991.

ESTEVES, F. A. Fundamentos da Limnologia. $2^{\text {a }}$ Ed Interciência Rio de Janeiro. 1998. 602 p.

FLORENZANO, T. G. Iniciação em Sensoriamento Remoto: Imagens de satélites para estudos ambientais. $2^{\text {a }}$ Ed.Oficina de Textos, São Paulo, 2007

FLORENZANO, T. G. Geotecnologias na Geografia Aplicada: difusão e acesso, Revista do Departamento de Geografia, USP no $17, \quad$ p. $24 \quad-\quad 29.2005$. https://doi.org/10.7154/RDG.2005.0017.0002.

IBGE. Instituto Brasileiro de Geografia e Estatística. Base de Informações do Censo Demográfico 2010: resultados do universo por setor censitário - documentação do arquivo. Rio de Janeiro: IBGE, 2011, 201 p.

IPARDES, Instituto Paranaense de Desenvolvimento Econômico e Social. Leituras regionais: Mesorregião Sudoeste Paranaense. Curitiba, BRDE, 2004, 139 p.

JENSEN, J. R. Sensoriamento Remoto do Ambiente: uma perspectiva em Recursos Terrestres. Traduação de José Carlos Neves Epiphanio. São José dos Campos, SP: Parênteses, 2009. 598 p.

MONTEIRO, C. A. de F.; MENDONÇA, F. Clima urbano. São Paulo. Contexto. 2003

MOREIRA, M., A. Fundamentos do Sensoriamento Remoto e metodologias de aplicação. 3a edição. Viçosa: UFV. 3a edição. 2005.

MOREIRA, M. A. Uso de imagens do Google Earth capturadas através do software stitch map e do TM/Ladsat-5 para mapeamento de lavouras cafeeiras - nova abordagem metodológica. XV Simpósio Brasileiro de Sensoriamento Remoto. Curitiba, p. 481488. 2011.

NASCIMENTO, I. J. de. Emprego de técnicas de sensoriamento remoto e de geoprocessamento na análise multitemporal do fenômeno de ilhas de calor no município de Goiânia-GO (1986/2010). 96f. Dissertação (Mestrado em Geografia) - Universidade Federal de Goiás, Goiânia, 2011.
NICHOL, J. E. A GIS-Based Approach to Microclimate Monitoring in Singapore's HighRise Housing Estates. Photogrammetric Engineering \& Remote Sensing, v.60, n. 10, p. 1225-1232, 1994.

NOVO, E. M. L. de M. Sensoriamento remoto: princípios e aplicações. 3a edição. São Paulo, Edgard Blucher, 2008.

OLIVEIRA, A. S. Influência da vegetação arbórea no microclima e uso de praças públicas. Cuiabá,. 2011.

PONZONI, F. J.; Shimabukuro, Y. E. Sensoriamento Remoto no Estudo da Vegetação. Editora Parêntese, São Paulo. 2010.

RIBEIRO, A. G. As Escalas do clima. Boletim de Geografia Teorética, Rio Claro, v. 23, p. 288-294, 1993.

SANTOS, J. S. dos. Campo térmico urbano e sua relação com o uso e cobertura do solo em cidade tropical úmida. Revista Brasileira de Geografia Física, Recife, v. 03, p. 540557 , 2012. https://doi.org/10.26848/rbgf.v5i3.232851.

SOARES FILHO, B. S. Modelagem da dinâmica da paisagem de uma região de fronteira de colonização amazônica. $299 f$. Tese (Doutorado), Escola Politécnica, Universidade de São Paulo. São Paulo, 1998.

SCHOTT, J. R.; GERACE, A.; RAQUENO, N.; IENTILUCCI, E.; RAQUENO, R.; LUNSFORD, A. W. Chasing the TIRS ghosts: calibrating the Landsat 8 thermal bands, Earth Observing Systems XIX, Califórnia, v. 9218, p. A1 - A20, 2014. https://doi.org/10.1117/12.2063236.

SOUZA, S. B. de; JÚNIOR, L. G. F. Relação entre temperatura de superfície terrestre, índices espectrais e classes de cobertura da terra no município de Goiânia (GO). Revista Espaço Geográfico em Análise. Vol. 26. Paraná, $\quad$ p. $\quad 75-99 . \quad 2012$. https://doi.org/10.5380/raega.v26i0.30151.

TURKMAN, M. A.; SILVA, G. L. Modelos Lineares Generalizados - da teoria à prática. Sociedade Portuguesa de Estatística, Lisboa. 2000.

USGS (United States Geological Survey). Using the USGS Landsat 8 Product. Disponível em:

https://landsat.usgs.gov/Landsat8_Using_Pro duct.php .Acesso em: 29.out.2014.

WENG, Q.; LU, D.; SHUBRING, J. Estimation of land surface temperature-vegetation abundance relationship for urban heat island studies. Remote Sensing of Environment. n. $\quad 89, \quad$ p. $\quad 467-483, \quad 2004$. https://doi.org/10.1016/j.rse.2003.11.005 